\title{
Editorials
}

\section{Why ARE MEXICAN DATA IMPORTANT?}

\author{
D Coulombier ${ }^{1}$, J Giesecke (Johan.Giesecke@ecdc.europa.eu) ${ }^{1}$ \\ 1. European Centre for Disease Prevention and Control, Stockholm, Sweden
}

This issue of Eurosurveillance contains an article by a French team on the transmission of the new influenza $A(\mathrm{H} 1 \mathrm{~N} 1)$ in Mexico, which uses published figures from the outbreak to estimate important parameters for transmission, among them the reproduction rate, $\mathrm{R}$ [1]. Such studies may have important implications for public health action in Europe.

\section{What is $\mathbf{R}$ ?}

The growth rate of an epidemic is determined by two factors: the number of new persons infected by each case and the time from start of infectiousness in one case to start of infectiousness in the secondary cases caused by him/her. The first factor is called 'reproduction rate' and is usually denoted $R$. If the disease is spreading in a population that is totally susceptible the term 'basic reproduction rate' (Ro) is used. $\mathrm{R}$ is the product of four terms: the risk of transmission in one single contact between an infectious and a susceptible person, the frequency of such contacts in the population, the duration of infectivity of a case, and the proportion of susceptibles in the population. If $R>1$ this means that each case infects more than one new person, and the outbreak is likely to continue. If $\mathrm{R}<1$ the outbreak will eventually die out, even if there may be a number of cases before that. The time from infectiousness in one case to infectiousness in his/her secondary cases is called 'generation time' (Tg) and is basically a biological constant, even if its exact value depends on how it is estimated.

Values for the factors that determine $\mathrm{R}$ can be calculated on the basis of scientific knowledge of the disease, its context of transmission, and the immunity status of the population. However, during an epidemic an $\mathrm{R}$ value usually has to be derived from the analysis of the epidemic curve or by the study of transmission chains.

Several studies have now tried to estimate R (or Ro) and Tg for the new influenza $A(\mathrm{H} 1 \mathrm{~N} 1)$ virus from Mexican data. In the one published in this issue of Eurosurveillance [1], the authors use one exponential fitting and one real-time estimation model to arrive at an estimate of $\mathrm{R}$ between 2.2 and 3.1. This is higher than the value found in an article in Science [2], which estimated Ro to be 1.4-1.6 using three models: one exponential fitting, one genetic analysis, and two standard SIR models for a confined outbreak in La Gloria. Another analysis of the minor genetic changes in the virus over time arrived at a Ro estimate of 1.16 [3].

Why is Ro important in public health?

The reproduction rate reflects effectiveness of transmission, and therefore has important implications for the efforts that public health authorities would have to make in implementing health measures aiming at containing or mitigating the outbreak.

For example, with a Ro of 1.16 , preventing $14 \%$ of cases will result in eventually interrupting transmission, while with a Ro of 3.1 , preventing $68 \%$ cases would be needed - assuming a total random mixing of contacts in the population.

\section{Why are Ro estimates so different for influenza?}

A few studies have tried to measure Ro for seasonal influenza [4], and found it to be in the order of 1.2 to 1.4 . However, for most of the seasonal strains, there is already some immunity in the population from past seasons, which lowers the reproduction rate (and it should thus really not be called Ro in this situation). For any epidemic of a disease that leads to immunity after infection the initial Ro will also be higher than the actual R at any later stage, since the proportion still susceptible in the population will decrease. It should also be realised that delayed reporting of cases will affect an estimate of $\mathrm{R}$; a problem that adheres to the study in this issue and the others cited above.

\section{What influences Ro?}

The risk of transmission in a contact when an infective meets a susceptible is basically a biological constant (even if it varies over the time course of the infection), as is the duration of infectiveness. However, frequency of contacts varies considerably between

\section{F I G U R E}

Daily reported cumulative number of cases in Mexico, Canada, USA, and EU/EFTA countries, outbreak of new influenza $\mathrm{A}(\mathrm{H} 1 \mathrm{N1})$, April-May 2009

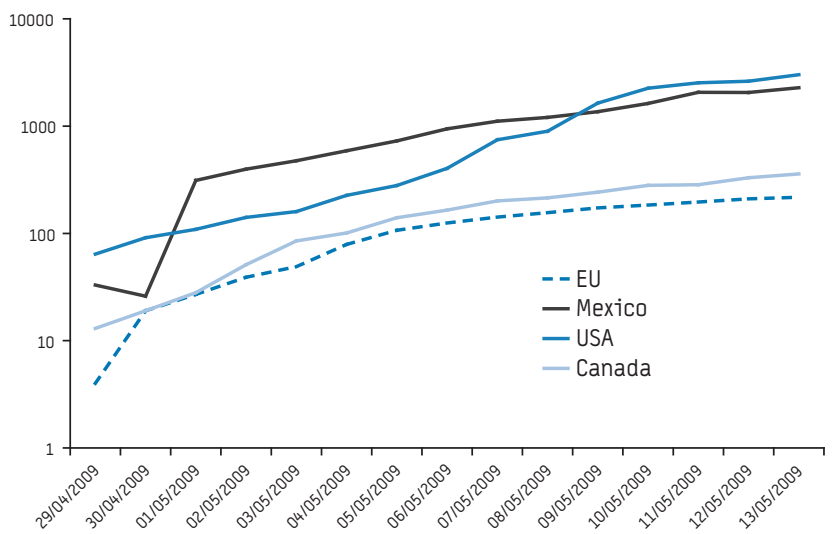


populations and population groups. For example, among children in schools or day care, the contact frequency is higher than among adults [5], and it also varies by culture, by family size in a society, by types of social interaction, etc.

\section{Why is the Ro from Mexico important?}

One could question why there is so much interest around studies of R and Ro based on Mexican data. Would they apply to Europe? One could guess that contact density might be higher in a Mexican setting, but on the other hand, since the epidemic has already run its course for some time there, the proportion of non-susceptibles would be higher in Mexico and the European situation would more approach a 'true' (higher) Ro, with a totally susceptible population.

In the graph, we have just compared the daily reported cumulative number of cases in Mexico, Canada, United States, and European Union and European Free Trade Association (EU/ EFTA) countries. On a semi-logarithmic scale it is evident that the slope for Europe is very much the same as for Mexico. It is difficult to estimate the time lag for Europe, but it seems that we are some 1-2 months behind. If the generation times are the same for both epidemics - which seems highly plausible - then an estimate of Ro for Mexico would apply also to Europe. A Ro just above 1 could mean that a containment strategy might be successful.

The European Centre for Disease Prevention and Control (ECDC) is continuously monitoring the situation and with more data being available every day in Europe we will obviously be able to have a better picture here soon as well. Nevertheless, the similarities of the shapes of the epidemics indicate that lessons from Mexico could apply also to Europe.

\section{References}

1. 1. Boëlle PY, Bernillon P, Desenclos JC. A preliminary estimation of the reproduction ratio for new influenza $A(\mathrm{H} 1 \mathrm{~N} 1)$ from the outbreak in Mexico, March-April 2009. Euro Surveill. 2009;14(19):pii=19205. Available from: http:// www.eurosurveillance.org/ViewArticle.aspx?ArticleId=19205

2. Fraser C, Donnelly CA, Cauchelmes S, Hanage WP, Van Kerkhove MD, Hollingsworth TD, et al. Pandemic potential of a strain of influenza A (H1N1): early findings. Published 11 May 2009 on Science Express. DOI: 10.1126/science.1176062. Available from: http://www.sciencemag.org/cgi/content/abstract/1176062

3. Rambaut A. Human/Swine A/H1N1 flu outbreak - BEAST analysis. Available from: http://tree.bio.ed.ac.uk/groups/influenza/wiki/178c5/BEAST_Analysis_29_ Apr_2008_-_Andrew_Rambaut.html

4. Chowell G, Miller MA, Viboud C. Seasonal influenza in the United States, France, and Australia: transmission and prospects for control. Epidemiol Infect. 2008;136(6):852-64.

5. Keeling MJ, Eames KT. Networks and epidemic models. J R Soc Interface. 2005;2(4):295-307.

This article was published on 14 May 2009.

Citation style for this article: Coulombier D, Giesecke J. Why are Mexican data important?. Euro Surveill. 2009:14(19):pij=19212. Available online: http://www. eurosurveillance.org/ViewArticle.aspx?ArticleId=19212 